\title{
Left atrial appendage closure: size does matter!
}

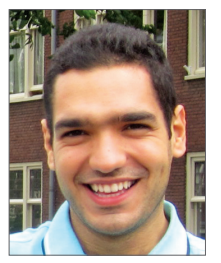

Guy Achkouty $^{1}$, MD; Adela Rohnean ${ }^{2}, \mathrm{MD}$; Christophe Caussin ${ }^{1}, \mathrm{MD}$;

Nicolas Amabile ${ }^{*}, \mathrm{MD}, \mathrm{PhD}$

1. Department of Cardiology, Institut Mutualiste Montsouris, Paris, France; 2. Department of Radiology, Institut Mutualiste Montsouris, Paris, France

This paper also includes supplementary data published online at: http://www.pcronline.com/eurointervention/128th_issue/258
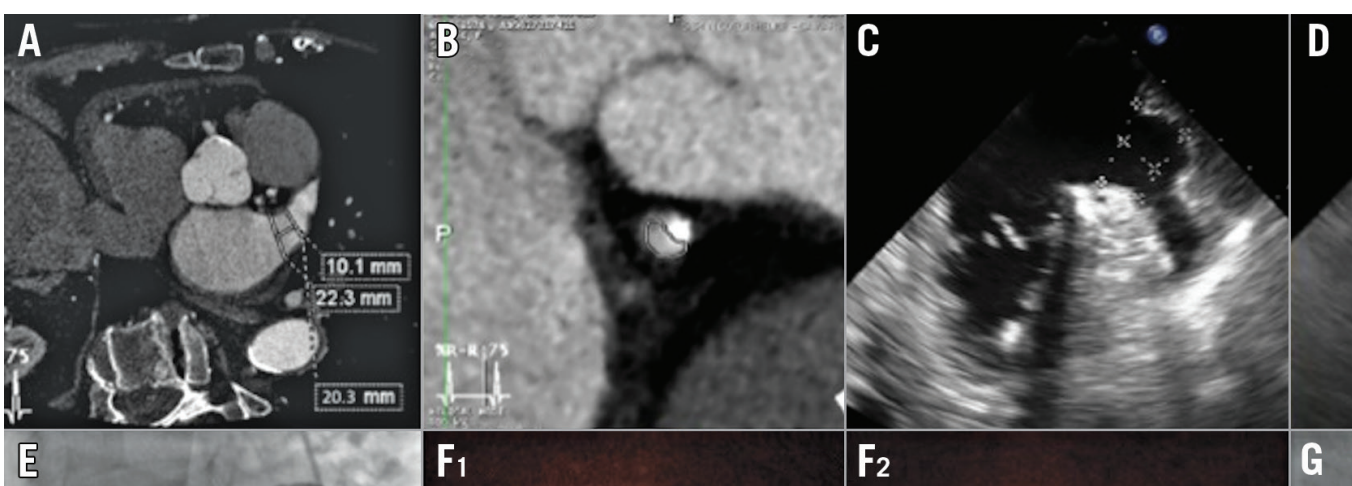

D
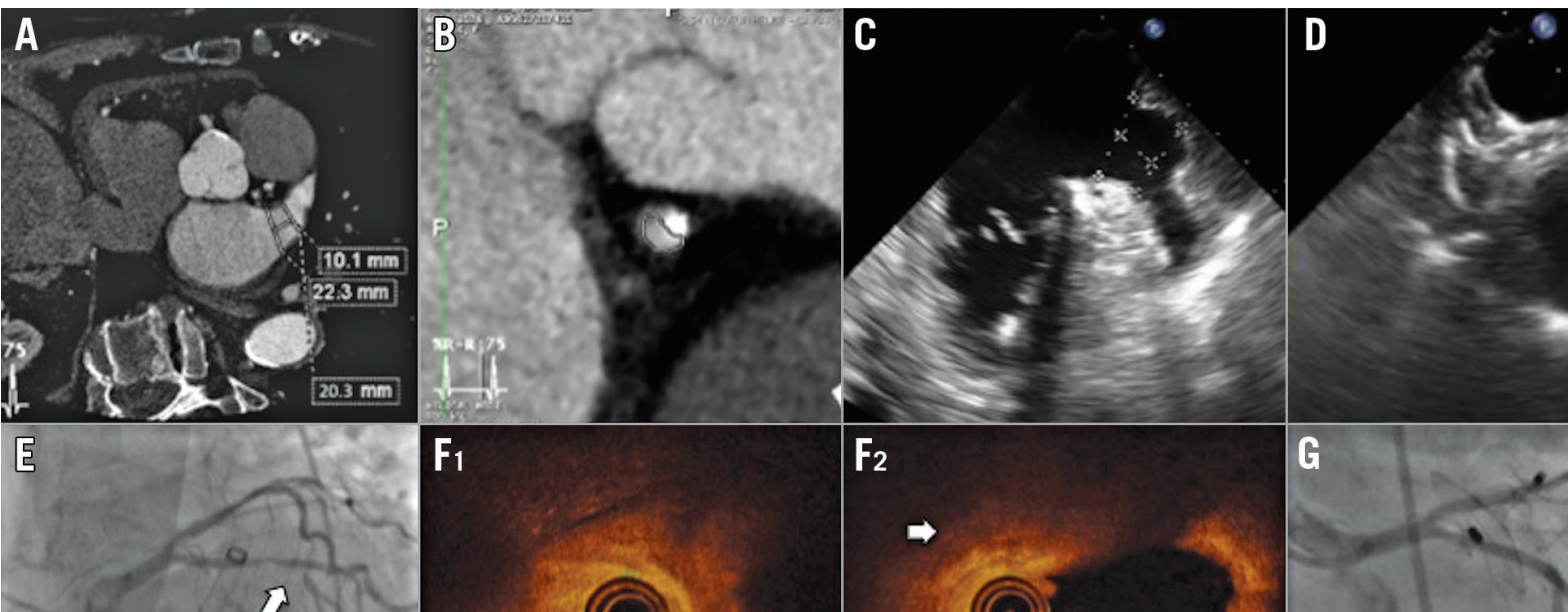

$\mathbf{F}_{1}$
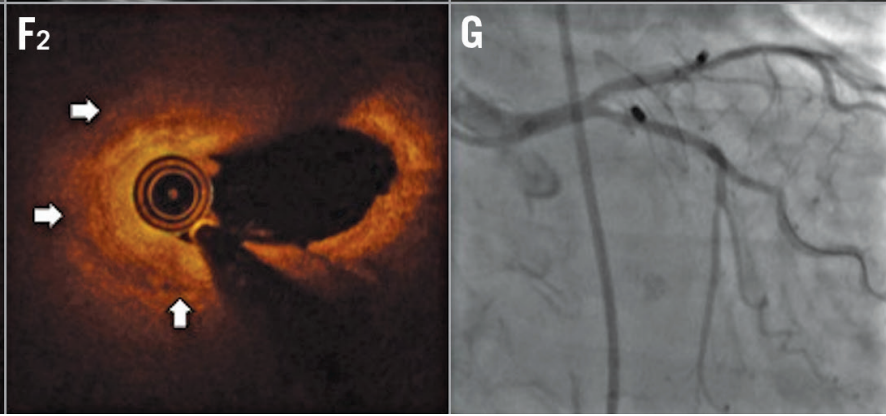

*Corresponding author: Department of Cardiology, Institut Mutualiste Montsouris, 42 Bd Jourdan, 75014 Paris, France. E-mail: nicolas.amabile@imm.fr 
An 86-year-old woman was referred to our institution for percutaneous left atrial appendage (LAA) occlusion. She was contraindicated for oral anticoagulation after a severe haemorrhagic shock related to diverticular disease. Her $\mathrm{CHA}_{2} \mathrm{DS}_{2}$-VASc and HASBLED scores were 6 and 4, respectively.

Preprocedural cardiac computed tomography (CT) scan confirmed the absence of thrombus in the LAA, the dimensions of which were measured to $22 \mathrm{~mm}$ in the neck (Panel A). An intermediate atherosclerotic plaque in the proximal circumflex artery was also noticed (Panel B). The procedure was performed under general anaesthesia and transoesophageal echocardiography (TEE) guidance. The LAA landing zone and ostium diameters were measured at 20 and $22 \mathrm{~mm}$, respectively, by TEE (Panel C), leading to the implantation of a $25 \mathrm{~mm}$ AMPLATZER ${ }^{\mathrm{TM}}$ Amulet $^{\mathrm{TM}}$ occluder (St. Jude Medical, St. Paul, MN, USA). After the device deployment, angiographic and echocardiographic control showed correct disc and lobe alignment and a severe lobe compression (Panel D). A new ST elevation in the inferolateral leads was identified on ECG monitoring. Therefore, the device was retrieved into the shaft. Emergent coronary angiography showed a severe stenosis of the proximal circumflex artery just beside the LAA (Panel E). ST segment resolution was observed only a few minutes after device retrieval and intracoronary nitrate injection. A smaller $22 \mathrm{~mm}$ AMPLATZER $^{\mathrm{TM}}$ Amulet $^{\mathrm{TM}}$ device was then implanted (Moving image 1) before treating the coronary lesion.

An optical coherence tomography (OCT) analysis of the circumflex artery showed an acute destabilisation of the initial circumflex lesion, related to extrinsic compression and putative plaque haemorrhage (Panel F1, Panel F2, Moving image 2). A $2.25 \times 24 \mathrm{~mm}$ everolimus-eluting stent (SYNERGYTM II; Boston Scientific, Marlborough, MA, USA) was then implanted with the help of specific bifurcation techniques (Panel G). The patient was discharged on dual antiplatelet therapy (aspirin and clopidogrel) for three months and the subsequent evolution was uneventful.

LAA occlusion procedures using the AMPLATZER ${ }^{\text {TM }}$ Amulet ${ }^{\mathrm{TM}}$ device lead to low numbers of major adverse events. Hence, the most recent data report limited incidence of death $(0.2 \%)$, major bleeding $(2.4 \%)$, tamponade $(1.2 \%)$, stroke $(0.2 \%)$, major vascular complications $(0.9 \%)$ or device embolisation $(0.1 \%)$ after implantation ${ }^{1}$. To the best of our knowledge, this is the first report of a circumflex mechanical compression by LAA occluder device leading to atherosclerotic plaque destabilisation documented with OCT.

This case illustrates the issues in adequate device size choice, despite multimodal imaging analysis. In our case, the most plausible mechanism leading to destabilisation appears to be arterial extrinsic compression by the device's lobe that induced plaque haemorrhage. Whether this complication could have been favoured by per-procedural anticoagulation remains unknown. Unfortunately, there is no recommendation regarding a systematic evaluation of the circumflex artery lumen and its intimate relation with the LAA before the procedure. This case advocates the cautious analysis of the pre-intervention cardiac CT scan.

\section{Conflict of interest statement}

N. Amabile and C. Caussin have received consulting fees from Abbott/St. Jude Medical. The other authors have no conflicts of interest to declare.

\section{Reference}

1. Landmesser U, Schmidt B, Nielsen-Kudsk JE, Chi Lam SC, Park JW, Tarantini G, Cruz-Gonzalez I, Geist V, Della Bella P, Colombo A, Zeus T, Omran H, Piorkowski C, Lund J, Tondo C, Hildick-Smith D. Left atrial appendage occlusion with the AMPLATZER Amulet device: periprocedural and early clinical/ echocardiographic data from a global prospective observational study. EuroIntervention. 2017;13:867-76.

\section{Supplementary data}

Moving image 1. $22 \mathrm{~mm}$ AMPLATZER Amulet device implantation.

Moving image 2. Pre-PCI circumflex artery OCT analysis.

The supplementary data are published online at:

http://www.pcronline.com/

eurointervention/128th issue/258 